\title{
The treatment of distal radius fractures
} accompanying dorsally displaced free fragments beyond the watershed line with turning radius and distal volaris radius system plate fixation via the distal palmar approach

\section{Zhaofeng Jia}

Shenzhen People's Hospital

Shijin Wang

Taian City Central Hospital

Wei Jiang

Shenzhen People's Hospital

Chuangli Li

Shenzhen People's Hospital

Jiandong Lin

Shenzhen People's Hospital

Qisong Liu

Shenzhen People's Hospital

Guangheng Li

Shenzhen People's Hospital

Xinjia Hu ( $\sim$ hxjys888@icloud.com)

\section{Research article}

Keywords: Distal radius fracture, Watershed line, Dorsal displaced, Fracture internal fixation, Distal volaris radius, Distal palmar approach, Radius turning

Posted Date: March 11th, 2020

DOI: https://doi.org/10.21203/rs.3.rs-16766/v1

License: (c) (i) This work is licensed under a Creative Commons Attribution 4.0 International License. Read Full License 
1 The treatment of distal radius fractures accompanying dorsally displaced free fragments beyond the

2 watershed line with turning radius and distal volaris radius system plate fixation via the distal

3 palmar approach

$4 \quad$ Running title:

5 A new technique for the treatment of distal radius fracture

6 Zhaofeng Jia ${ }^{1}$, Shijin Wang ${ }^{2}$, Wei Jiang ${ }^{1}$, Chuangli Li ${ }^{1}$, Jiandong Lin ${ }^{1}$, Qisong Liu ${ }^{1}$, Guangheng Li $^{1}$,

$7 \quad$ Xinjia $\mathrm{Hu}^{1 *}$

$8 \quad$ Zhaofeng Jia

9 labidaxin2016@163.com

10 Shijin Wang

11 wangshijin1314@163.com

12 Wei Jiang

13 docjw@sina.com

14 Chuangli Li

$151040283781 @ q q . c o m$

16 Jiandong Lin

17 1037188943@qq.com

$18 \quad$ Qisong Liu

19 liuqizong123@126.com

20 Guangheng Li

21 liguangheng@hotmail.com

22 Xinjia Hu

23 hxjys888@icloud.com 
25 Abstract

26 Background: Although distal radius fractures (DRFs) are clinically common, DRFs accompanied by 27 dorsally displaced free fragments beyond the watershed line are much less so. At present, it is very 28 difficult to fix and stabilize the displaced free fragments far away from the watershed line with a plate.

29 Our aim was to investigate the clinical effect of DRFs with distally displaced dorsal free mass treated 30 with distal volaris radius (DVR) combined with turning of the radius via the distal palmar approach. 
Conclusion: Treatment of distal radius fractures with accompanying dorsally displaced free fragments

49 beyond the watershed line with turning of the radius and the DVR plate system via the distal palmar 50 approach is effective and has no obvious complications.

51 Keywords: Distal radius fracture, Watershed line, Dorsal displaced, Fracture internal fixation, Distal volaris radius, Distal palmar approach, Radius turning

\section{Introduction}

Distal radius fractures (DRFs) are common fractures in adults, usually caused by high-energy trauma [1]. High-energy injuries often lead to DRFs with dorsally or volarly displaced free fracture fragments; watershed line is very rare, making volarly locking plate fixation difficult. This kind of fracture belongs to the class of intra-articular fractures, which require anatomical reduction and firm fixation. If the

60 fixation is not stable enough, the fracture may be displaced and even undergo subluxation, which may 61 lead to malunion or non-union of the fracture, as well as early onset of traumatic arthritis and impaired wrist function. So far, no consensus has been reached on the definition of this type of injury, its mechanism or optimal treatment protocols [2-4]. Tendon irritation and even serious complications,

64 including tendon rupture, can easily occur when the plate is fixed via the dorsal approach. The 65 reduction and fixation effect of the palmar combined dorsal approach was beneficial, but this technique 66 can cause severe surgical trauma. External fixation with stents does not properly correct and fix the 67 displaced dorsal fracture fragments. Therefore, no current treatments are sufficient to adequately 68 resolve these fractures, making them a challenge for orthopedic surgeons $[5,6]$.

69 In recent years, the introduction of the DVR anatomical palmar plate system has revolutionized 
treatment of distal radius fractures [7]. The DVR plate system is direct and easy to operate, allowing

71 patients to quickly regain wrist movement [8]. The excellent biomechanical strength of the DVR plate

72 is based on its ability to provide a three-dimensional scaffold structure and strong subchondral support

73 for the articular surface of the distal radius. It locks the screw and screw structure by the fixed angle

74 formed by the cross arrangement of two rows of screws at its far end. The role of these screws is to

75 stabilize the subchondral bone and reduce disturbance to the dorsal tendon. The pull screw can fix the

76 dorsally displaced fracture fragments and pull them to the radius, so as to resolve the fracture [9-11].

77 Although DVR plates are increasingly used in the treatment of distal radius fractures, it is difficult to

78 fix a distal radius fracture with dorsal carpal displacement and free fragments by DVR anatomical volar

79 plate alone. However, incorporating a dorsal incision to reduce the bone fragments with dorsal

80 displacement would result in excessive surgical trauma. Therefore, we innovatively employ the volar

81 approach to separate the distal radius and turn it to the volar side. After this, we could directly observe

82 the displaced fracture on the dorsal side. Then, we use a Kirschner wire to temporarily fix the fracture

83 block before further stabilizing and fixing it through the DVR plate. After these procedures, we could

84 successfully repair the fracture of the distal radius beyond the watershed line with the displaced fracture

85 block on the dorsal side.

86 The purpose of this study is to summarize the surgical method of the distal palmar approach combined

87 with DVR plate fixation after radius turnover for the treatment of distal radius fractures accompanied

88 by dorsally displaced free fragments beyond the watershed line, as well as to evaluate the therapeutic

89 effects of this technique.

90

$91 \quad$ Materials and methods

92 General information 
93 This study was approved by the Ethics Committee of Shenzhen People's Hospital at Jinan University.

94 All volunteers gave informed consent prior to participating in the study.

95 From 2015 to 2019, 25 patients with distal radius fractures accompanied by dorsally displaced free 96 fragments beyond the watershed line were included in our study. Fifteen of the patients got their 97 fractures from accidental fall, and 10 received their injuries from traffic accidents. All of the patients 98 had closed fresh fractures. The study cohort included 14 males and 11 females with an average age of 9934.5 years (21-50 years). According to AO classifications, all patients had type C fractures (Table 1), as 100 determined by the presence of articular surface crushing, a fracture line that spread beyond the 101 watershed line and the presence of dorsally displaced fracture fragments (Figure 1). The patients were 102 first treated with plaster external fixation and detumescence. The average operation time was 68.25 103 minutes (42-78 minutes), and the operation occurred an average of 4.5 days after injury (ranging from 104 0-7 days). The average follow-up time was 12.5 months (ranging from 8 to 22 months) (Table 2). 

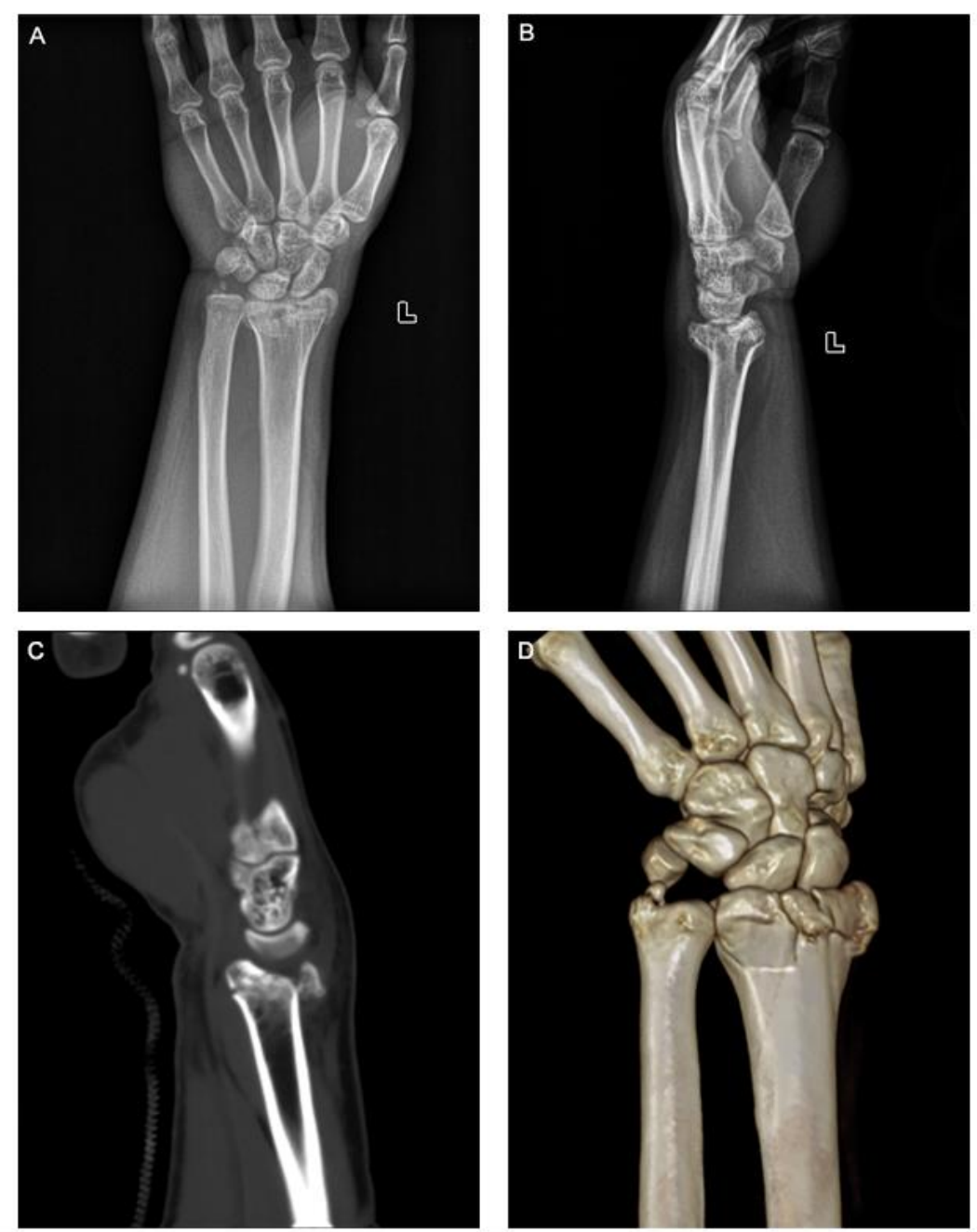

106 Figure 1. A 42-year-old man suffered from a comminuted fracture of the left distal radius after falling

107 from a high place. A dorsal free fracture fragment is shown at the distal radius. (A, B) Anteroposterior 108 and lateral X-rays before surgery. (C, D) Computed tomography of the fracture.

110 Table 1. Fracture type classification of the patients in our study by AO Classification ${ }^{[12]}$

\begin{tabular}{lcl}
\hline AO Classification(2R3-) & Number of Patients & \\
\hline C 3.1 & 15 & Type C (complete articular) \\
C 3.2 & 8 & \\
C 3.3 & 2 & \\
\hline
\end{tabular}


115 Table 2. Demographics and clinical characteristics of patients

\begin{tabular}{lc}
\hline Variable & Value (mean value) \\
\hline Patient & 25 \\
Sex (male: female) & $14: 11$ \\
Mean age (yr) & 34.5 \\
Smoker & 3 \\
Time to surgery (day) & 4.5 \\
Operation time (min) & 68.25 \\
Follow-up period (months) & 12.5 \\
\hline
\end{tabular}

\section{Surgical technique}

118 After anesthesia, an $8 \mathrm{~cm}$ long incision was made along the flexor carpi radials (FCR). The skin, 119 subcutaneous fascia and deep fascia were consecutively cut open, and the FCR was pulled toward the 120 ulnar side. The median nerve was protected at the same time. The FCR was moved distally until it 121 reached the level of the scaphoid node in order to enlarge the space under the palmar tendon of the 122 forearm and expose the pronator anterior muscle. After these manipulations, we were able to touch the 123 distal radius and identify the palmar margin of the lunate fossa. We then marked both the watershed 124 line and the proximal end of the lateral radius with sterile marker pen for later procedures. We used a 125 sharp scalpel to cut both the proximal end of the lateral edge of the radius and the overlying ridge line, 126 release the pronator muscle, cut off the middle support belt from the watershed line, peel off and 127 expose the brachioradialis muscle stop, and obliquely disconnect it. Then, we turned the proximal 128 segment of the radius inward, peeled and exposed the distal articular surface of the radius, restored the 129 distal articular surface under direct vision and turned the proximal segment back after confirming 130 satisfactory recovery of the articular surface. We placed the DVR (Zimmer-Biomet) plate on the palmar 131 side. The distal end of the plate was aligned with the proximal side of the watershed line. A Kirschner 
132 pin was inserted into the medial Kirschner pin hole of the proximal row of pin holes to fix the distal 133 bone block. The correct position of the plate was determined by C-arm fluoroscopy lateral film and 134 oblique lateral film (inclined $20-25^{\circ}$ ). At this angle, the gram needle should be $2.0-3.0 \mathrm{~mm}$ below the 135 articular cartilage. In order to stabilize the lunar fossa, a $2.0 \mathrm{~mm}$ drill bit was used to drill from the 136 ulnar side through a disposable quick guide. The length of the near row of screws was obtained by 137 reading the scale of the round surface on the sounder. After confirming the drilling depth, the quick 138 guide was removed with a screwdriver. Then, we used the same screwdriver to insert screws of the 139 correct length into all distal pin holes, and cortical screws were screwed into the remaining proximal 140 screw holes. After pulling out the temporary fixing Kirschner wire, fracture recovery was satisfactory 141 based on the fluoroscopy observation, and there were no obvious abnormalities in wrist joint movement. 142 After hemostasis, the brachioradialis tendon and the pronator were sutured, and the incision was closed 143 (Figure 2). All operations were performed by an experienced orthopedic surgeon.
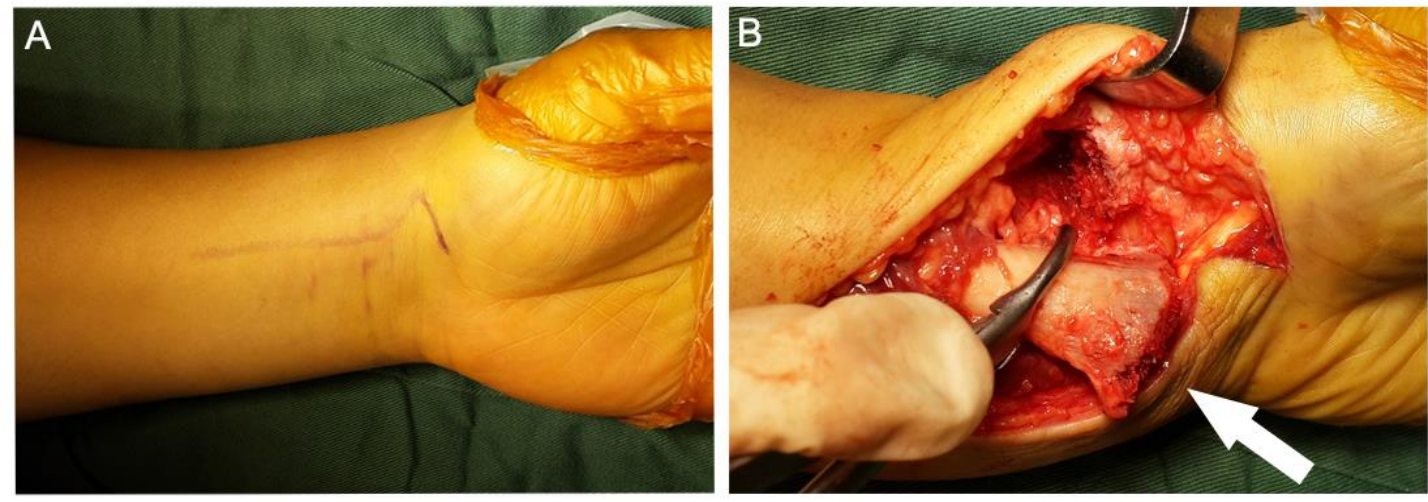

145 Figure 2. The incision and distal radius were reversed during the operation. (A) A zigzag incision was made across the wrist flexion crease to allow for better access and visualization. (B) Intra-focal exposure was obtained by pronating the proximal fragment out of the way with the turning radius (marked by the white arrow) using a bone clamp.

\section{Postoperative care}


151 After anesthesia subsided, all patients began to stretch and flex their wrists slightly and move their

152 elbows freely without bearing weight or participating in distance exercise or heavy physical activity of 153 the affected limbs. Patients were suggested to be cautious about twisting exercises within three months 154 of the operation.

156 Radiological outcome

157 Preoperative X-ray and three-dimensional computed tomographic (CT) images were used to analyze 158 the fracture types and observe the dorsally displaced fracture fragments far from the watershed line. 159 X-ray films of anteroposterior and lateral carpal joints were taken immediately after the operation 160 (Figure 3).

161 At the end of the follow-up, X-ray was used to confirm fracture healing, distal radial height, volar tilt, 162 and radial inclination, as well as to assess any evidence of traumatic arthritis (Figure 4). Radiologic 163 measurements were performed by two orthopedic doctors and a radiologist. 


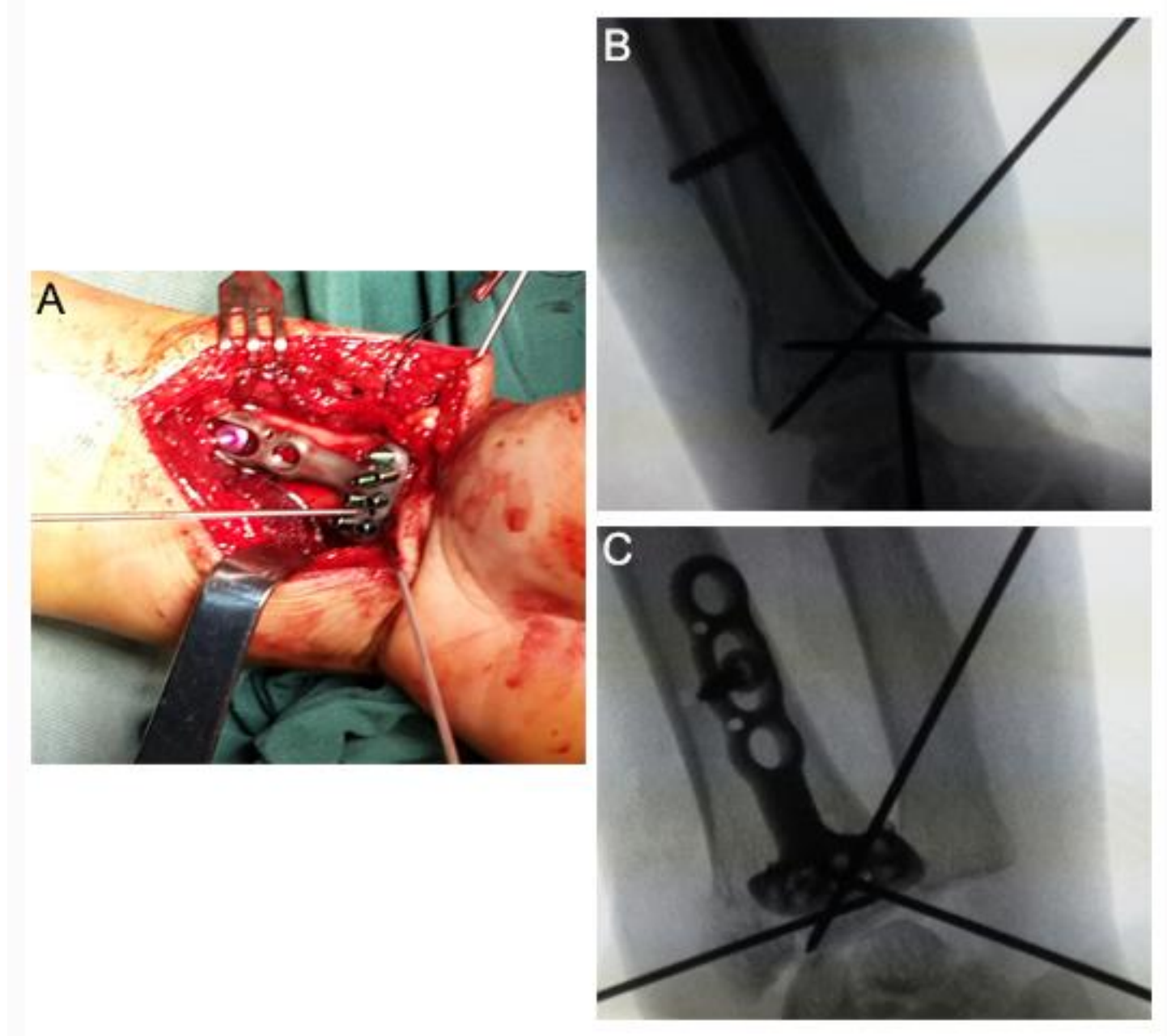

166 Figure 3. Image of the operation and fluoroscopy observation. (A) Intraoperative image after the 167 placement of DVR with K-wires after open reduction of the fracture. (B, C). Fracture temporally fixed 168 with two K-wires and plate temporally fixed with another K-wire (B, Lateral view; C, Anteroposterior 169 view).

\section{Clinical evaluation}

172 At the end of the follow-up, wrist function -- including ROM, MMWs and DASH -- was evaluated.

173 ROM (dorsiflexion, volar flexion, ulnar deviation and radial deviation) of the wrist and forearm were

174 measured with a goniometer. Pain visual analogue scale (VAS) was also evaluated. 
177 During the follow-up, we monitored possible postoperative complications, including wound infection, 178 non-unions, malunions, tendon rupture, traumatic osteoarthritis, joint mobility disorders, persistent 179 neuropathy and complex regional pain syndrome.

$181 \quad$ Statistical analysis

182 Statistical analysis was performed using the SPSS statistical version 19.0 in this study. Data was shown 183 as mean \pm standard deviation $(\mathrm{x} \pm \mathrm{s})$. The student $t$ test was used for continuous variables. P-values less 184 than 0.05 were regarded as statistically significant different.

187 All patients had satisfactory postoperative wound healing, and all patients showed evidence of bone 188 healing during the follow-up. Functional recovery was satisfactory without any obvious complications. 189 At the end of the follow-up, mean radial height was $10.5 \mathrm{~mm}$ (ranging from 8.1 to $12.6 \mathrm{~mm}$ ), mean 190 volar tilt angle was $9.28^{\circ}$ (ranging from 5.7 to $12.8^{\circ}$ ) and mean radial inclination angle was $23.02^{\circ}$ 191 (ranging from 19.5 to $29.3^{\circ}$ ). There were no signs of post-traumatic arthritis based on patient 192 radiography (Table 3) (Figure 4).

193 Table 3. Radiographic evaluation of the fracture after surgery

\begin{tabular}{cc}
\hline Variable & Mean (range) \\
\hline Radial height $(\mathrm{mm})$ & $10.5(8.1-12.6)$ \\
Volar tilt $\left(^{\circ}\right)$ & $9.28\left(5.7^{\circ}-12.8\right)$ \\
Radial inclination $\left(^{\circ}\right)$ & $23.02(19.5-29.3)$ \\
\end{tabular}



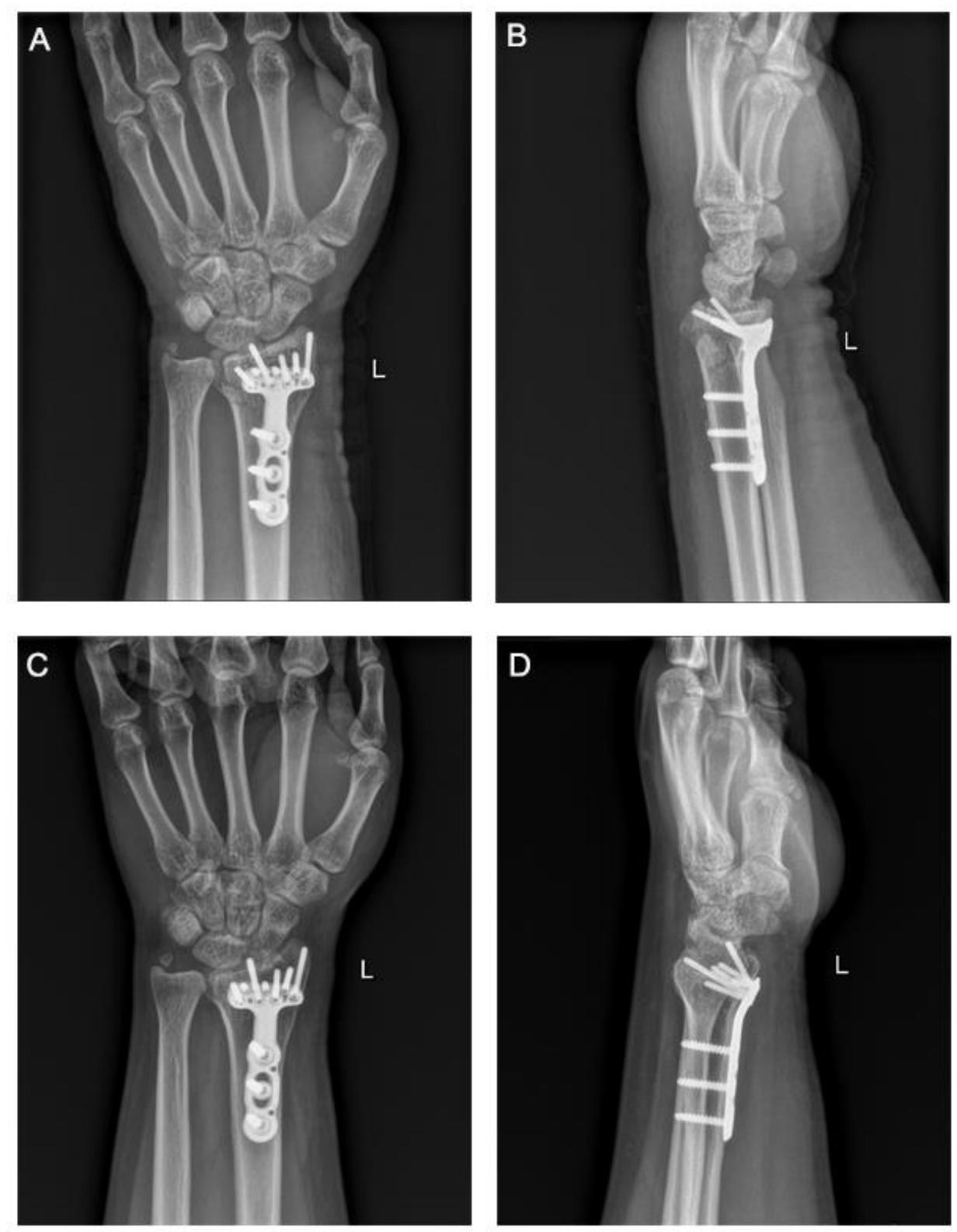

196 Figure 4. Radiographical images after the operation. (A, B) Anteroposterior (A) and lateral (B) 197 radiographs showing that the alignment of the fracture and position of the plate were satisfactory on 198 postoperative day 1. (C, D) Anteroposterior (C) and lateral (D) radiographs at twelve months 199 post-operation, showing that the left wrist has completed bony union.

200 All patients achieved satisfactory recovery and bony union. Wounds healed appropriately, and function 201 recovered well without any obvious complications. At the end of the follow-up, the mean MMWS 202 across all patients was $78.8^{\circ}$ (ranging from 61 to $90^{\circ}$ ), and the mean DASH score was 16.25 (ranging 203 from 11 to 21 ). The mean dorsiflexion ROM was $77.1^{\circ}$ (ranging from 59 to $83^{\circ}$ ), and the mean volar 
204 flexion $\mathrm{ROM}$ was $76.5^{\circ}$ (ranging from 62 to $81^{\circ}$ ). The average ROM of ulnar deviation angle was $21.4^{\circ}$

205 (ranging from 15 to $28^{\circ}$ ), the average ROM of radial deviation angle was $17.5^{\circ}$ (ranging from 12 to $23^{\circ}$ )

206 and the average VAS score of pain was 1.4 (ranging from 1 to 3). (Table 4 and Figure 5)

207

208 Table 4. Clinical results

\begin{tabular}{cc|}
\hline Score & Mean (range) \\
\hline Dorsiflexion $\left(^{\circ}\right)$ & $77.1(59-83)$ \\
Volar flexion $\left(^{\circ}\right)$ & $76.5(62-81)$ \\
Ulnar deviation $\left(^{\circ}\right)$ & $21.4(15-28)$ \\
Radial deviation $\left(^{\circ}\right)$ & $17.5(12-23)$ \\
Modified Mayo wrist score & $78.8(61-90)$ \\
DASH score & $16.25(11-21)$ \\
VAS & $1.4(1-3)$ \\
\hline
\end{tabular}



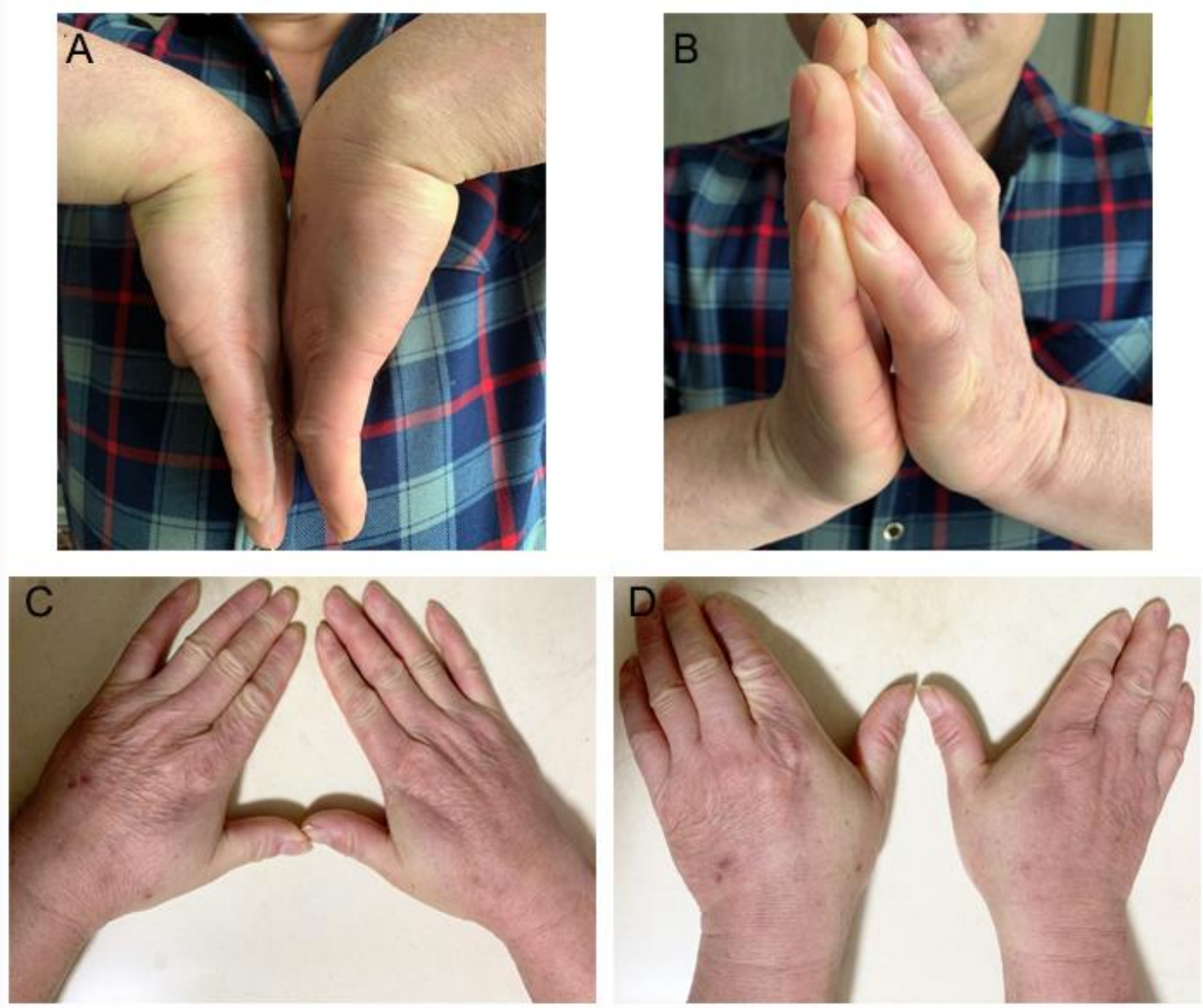

211 Figure 5. Excellent clinical results 12 months after surgery. The patient achieved volar flexion of $77^{\circ}$

212 (A), dorsal flexion of $80^{\circ}(\mathbf{B})$, radial deviation of $20^{\circ}(\mathbf{C})$ and ulnar deviation of $23^{\circ}(\mathbf{D})$.

\section{Discussion}

215 It is difficult for orthopedic surgeons to deal with complex fractures, including distal radius fractures

216 accompanied by dorsal free fragments beyond the watershed line. Few studies for the treatment of this

217 complex fracture have been reported, because a general locking plate cannot effectively fix the distal

218 free fracture fragments $[13,14]$.

219 The anatomical volar locking plate system of the distal radius of DVR is a new internal fixation system

220 that combines the advantages of previously existing internal fixation systems. First, the design of the

221 plate is highly matched with the anatomic structure of the distal radius. The distal shape of the plate is

222 well-matched to the "watershed line" and surface anatomical morphology of the distal radius on the 
volar side. Selvan et al. believe that DVR can reduce friction between the plate and tendon, more so than any other distal radius plate [15]. When the plate is placed at the farthest end, it can effectively prevent tendon irritation, thus reducing disturbance to the soft tissue. Second, Vanhaecke et al. believe that the key to this technology's success is its ability to obtain solid fixation and perfect subchondral support [16]. The DVR plate fits the "Watershed line" of the distal radius on the volar side, providing strong support for volar marginal bone mass. The locking support rod and screw provide a solid nail/plate interface. The DVR system, consisting of two rows of 7 fixation screws and a patented three-dimensional scaffold generated by intersecting screws, has an improved supporting effect on the comminuted fracture and will not cause bone collapse. Third, the placement of a DVR plate is simple, leading to increased accuracy and decreased operation time. The DVR system is designed to use the "watershed line" on the palmar side of the distal radius as a natural anatomic mark and is placed by pushing the plate to the "watershed line," resulting in an improved fit between the plate and bone surface and preventing the screw from entering the joint surface. Keeping screws out of the joint surface is one of the key steps for successful internal fixation.

Based on 187 cases with an average follow-up period of 2.5 years, Macfarlane et al. found that $8 \%$ of complications were caused by metacarpal plate screws, including three cases of tendon injury. This led them to recommended DVR for treatment of unstable distal radius fractures [17]. Two types of locking screws are used in the DVR system, threaded and unthreaded. Through mechanical experiments with a cadaver radius, Martineau et al. confirmed that there is no obvious difference between these two kinds of screws. However, they additionally concluded that the smooth rod screw is convenient to use and will not cause fracture block rotation [18]. Several reports have shown that the effect of DVR on anatomical structure and wrist function was satisfactory after the operation based on a 3-month follow-up [19, 20]. For fractures far from the distal radius watershed, many researchers and doctors 
tend to use an external fixation stent for fixation, as they believe that it is difficult to fix the plate

247 effectively. However, Jorge Mora et al. reported that the occurrence of complications from an external

248 fixation stent is significantly higher than that from the DVR system [21].

249 With a single volar incision, it is difficult to reduce and fix the displaced fracture block. To solve this

250 problem, people have tried to reduce the displaced fracture block using manual traction or to add a

251 dorsal incision to assist in reduction of the displaced fracture block [22-25]. In the present study, we cut

252 off the brachioradialis muscle, turned the proximal radius over and completely exposed the distal dorsal

253 fracture block and joint surface. Under this direct vision, we could recover the distal joint surface

254 through first treating the dorsal joint surface, then the metacarpal joint surface, before finally turning

255 the proximal radius over. By using these procedures, we could complete the operation on both sides of

256 the metacarpal dorsal side with a single volar incision. After the operation, all wounds healed and wrist

257 function was satisfactorily recovered.

258 However, we believe that not all distal radial fractures require radial turnover. In our opinion, the 259 indications for radial turnover are as follows: cases with severe crushing of the articular surface; cases 260 requiring dorsal fracture block reduction but presenting difficulties for dorsal incision. The 261 contraindications for radial turnover are as follows: cases without the presence of serious joint surface 262 crushing; cases with large distal metacarpal fractures. If the fracture on the articular surface is not 263 serious, radius turnover is not required. In cases with large palmar fracture block, radius turnover is not 264 worthwhile, as it is difficult to directly view the dorsal fracture block even after the turnover.

265 Our study has several limitations. First of all, as the chosen surgical approach is determined by both the 266 type of fracture and the preference of the surgeon, this report reflects only the experiences of the 267 surgeons in this study. Secondly, the case number in our study is small, and there is no control group. 268 Compounded by the dearth of reports about the treatment of these complex fractures, we need more 
269 data to draw a solid conclusion. Moreover, we think that a longer follow-up period is needed to 270 accurately evaluate the occurrence of traumatic osteoarthritis.

272 Conclusion

273 For complex distal radius fractures accompanied by dorsally displaced free fragments beyond the 274 watershed line, the distal palmar approach to the distal radius, with turning radius technology and DVR 275 fixation, provides an excellent treatment option without trauma or obvious surgical complications. This 276 surgical technique is simple and easy to employ, giving it great promise for clinical application.

\section{Abbreviation}

279 DRFs: distal radius fractures; DVR: distal volaris radius; ROM: Range of motion; MMWS: Modified 280 mayo wrist score; DASH: Disabilities of Arm, Shoulder and Hand.

\section{Acknowledgements}

283 We thank the members of our research group. All authors have approved the final version of the 284 manuscript and read the journal's authorship agreement.

\section{Authors' contributions}

287 ZFJ did the surgery, collected the data, analyzed the data, drafted the manuscript, and carried out the 288 follow-ups. GHL supervised the project and reviewed the manuscript. WJ, CLL and JDL conceived of 289 the study, participated in its design and coordination, and helped to draft the manuscript. XJH was 290 responsible for the whole project, designed the study and supervised the study. All authors read and 291 approved the final manuscript. 


\section{Funding}

294 Natural Science Foundation of China (grant number: 81902196), the fund of the young and 295 middle-aged scientific research backbone cultivation project of Shenzhen People's Hospital (grant 296 number: SYKYPY201903).

298 Availability of data and materials

299 All the data and materials can be found in the manuscript.

301 Ethics approval and consent to participate

302 The study was approved by the ethics committee at Shenzhen People's Hospital and was conducted in 303 accordance with the Protocol of Helsinki. Informed consent was signed by the relatives of the patients.

\section{Consent for publication}

306 All individual persons consented for their data to be published.

\section{Competing interests}

309 The authors declare that they have no competing interests.

\section{Author details}

$312{ }^{1}$ Department of Osteoarthropathy, Shenzhen People's Hospital, The Second Clinical Medical College of 313 Jinan University and the First Affilliated Hospital of Southern University of Science and Technology, 314 Shenzhen, 518035, Guangdong Province, China 
$315{ }^{2}$ Department of Orthopaedics, Taian City Central Hospital, Shan dong Province, 271000, China

317 Reference

318 1. Chen NC, Jupiter JB. Management of distal radial fractures. J Bone Joint Surg Am. 2007 Sep; 319 89(9):2051e62.

320 2. Ng CY, McQueen MM. What are the radiological predictors of functional outcome following 321 fractures of the distal radius, J Bone Joint Surg Br. 2011;93(2):145-50.

3. Chang HC, Poh SY, Seah SC, Chua DT, Cha BK, Low CO. Fragment-specific fracture fixation and doublecolumn plating of unstable distal radial fractures using $\mathrm{AO}$ mini-fragment implants and Kirschner wires. Injury. 2007;38(11):1259-67.

4. Musgrave DS, Idler RS. Volar fixation of dorsally displaced distal radius fractures using the 2.4-mm locking compression plates. J Hand Surg. 2005;30(4):743-9.

5. Kachooei AR, Tarabochia M, Jupiter JB. Distal radius volar rim fracture fixation using DePuy-Synthes volar rim plate. J Wrist Surg. 2016;5(1):2-8.

6. Goorens CK, Geeurickx S, Wernaers P, Staelens B, Scheerlinck T, Goubau J. Midterm follow-up of treating volar marginal rim fractures with variable angle lcp volar rim distal radius plates. J Hand Surg Asian Pac Vol. 2017;22(2):184-7.

7. Chung KC, Watt AJ, Kotsis SV, Margaliot Z, Haase SC, Kim HM. Treatment of unstable distal radial fractures with the volar locking plating system. J Bone Joint Surg Am. 2006;88(12):2687-94.

8. Orbay JL, Fernandez DL. Volar fixed-angle plate fixation for unstable distal radius fractures in the elderly patient. J Hand Surg Am. 2004 Jan;29(1):96-102.

9. Frattini M, Soncini G, Corradi M, Panno B, Tocco S, Pogliacomi F. Complex fractures of the distal radius treated with angular stability plates. Chir Organi Mov. 2009;93:155-62. 
10. Sobky K, Baldini T, Thomas K, Bach J, Williams A, Wolf JM. Biomechanical comparison of different volar fracture fixation plates for distal radius fractures. Hand. 2008;3(2):96-101.

11. Willis AA, Kutsumi K, Zobitz ME, Cooney WP 3rd. Internal fixation of dorsally displaced fractures of the distal part of the radius. A biomechanical analysis of volar plate fracture stability. J Bone Joint Surg Am.2006;88(11):2411-7.

12. Audigé L, Slongo T, Lutz N, Blumenthal A, Joeris A. The AO pediatric comprehensive classification of long bone fractures (PCCF). Acta Orthop. 2017 Apr;88(2):133-9.

13. Iba K, Ozasa Y, Wada T, Kamiya T, Yamashita T, Aoki M. Efficacy of radial styloid targeting screws in volar plate fixation of intra-articular distal radial fractures: a biomechanical study in a cadaver fracture model. J Orthop Surg Res. 2010 Dec;5(1):90.

14. Eglseder WA. Distal radius fractures. J South Orthop Assoc. 1997 Winter;6(4):289-97.

15. Omokawa S, Abe Y, Imatani J, Moritomo H, Suzuki D, Onishi T. Treatment of intra-articular distal radius fractures. Hand Clin. 2017;33(3):529-43.

16. Vanhaecke J, Fernandez DL. DVR plating of distal radius fractures. Injury. 2015 Nov;46 Suppl 5:S33-6.

17. MacFarlane RJ, Miller D, Wilson L, et al. Functional outcome and complications at 2.5 Years following volar locking plate fixation of distal radius fractures. J Hand Microsurg. 2015 Jun;7(1):18-24.

18. Martineau D, Shorez J, Beran C, et al. Biomechanical performance of variable and fixed angle locked volar plates for the dorsally comminuted distal radius. Iowa Orthop J. 2014;34:123-28.

19. Yao J, Park MJ, Patel CS. Biomechanical comparison of volar locked plate constructs using smooth and threaded locking pegs. Orthopedics. 2014;37(2):e169.

20. Patel S, Menndez PB, Hossain FS, et al. Does the DVR plate restore bony anatomy following distal 
361 radius fractures? Ann R Coll Surg Engl. 2014;96(1):49-54.

362 21. Jorge-Mora AA, Cecilia-López D, Rodríguez-Vega V, et al. Comparison between external fixators 363 and fixed-angle volar-locking plates in the treatment of distal radius fractures. J Hand Microsurg. 2012 Dec;4(2):50-4.

365 22. Loveridge J, Ahearn N, Gee C, Pearson D, Sivaloganathan S, Bhatia R. Treatment of distal radial 366 fractures with the DVR-A plate--the early Bristol experience. Hand Surg. 2013;18(2):159-67.

367 23. Phillips AR, Al-Shawi A. Optimal positioning of the DVR plate in distal radius fractures: a 368 cadaveric examination of a referencing technique. Injury. 2012 Feb;43(2):209-12.

369 24. Frattini M, Soncini G, Corradi M, Panno B, Tocco S, Pogliacomi F. Complex fractures of the distal 370 radius treated with angular stability plates. Chir Organi Mov. 2009 Dec;93(3):155-62.

371 25. Tarallo L, Adani R, Mugnai R, Catani F. The treatment of distal radius articular fractures of C1-C2 372 type with DVR plate: analysis of 40 cases. Musculoskelet Surg. 2011 Dec;95(3):225-30. 
Figures
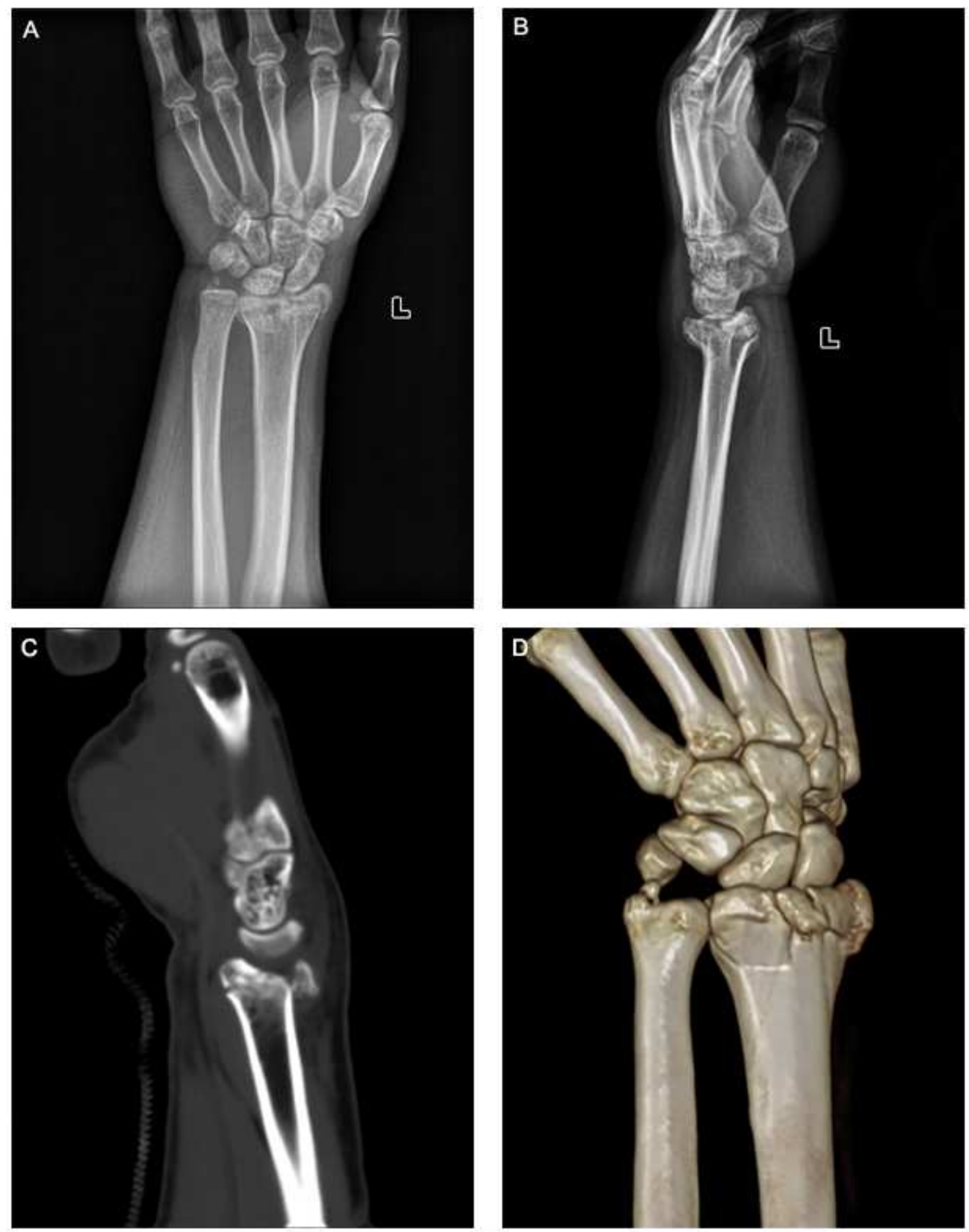

\section{Figure 1}

A 42 year old man suffered from a comminuted fracture of the left distal radius after falling from a high place. A dorsal free fracture fragment is shown at the distal radius. A, B ) Anteroposterior and lateral $X$ rays before surgery. ( C, D ) Computed tomography of the 

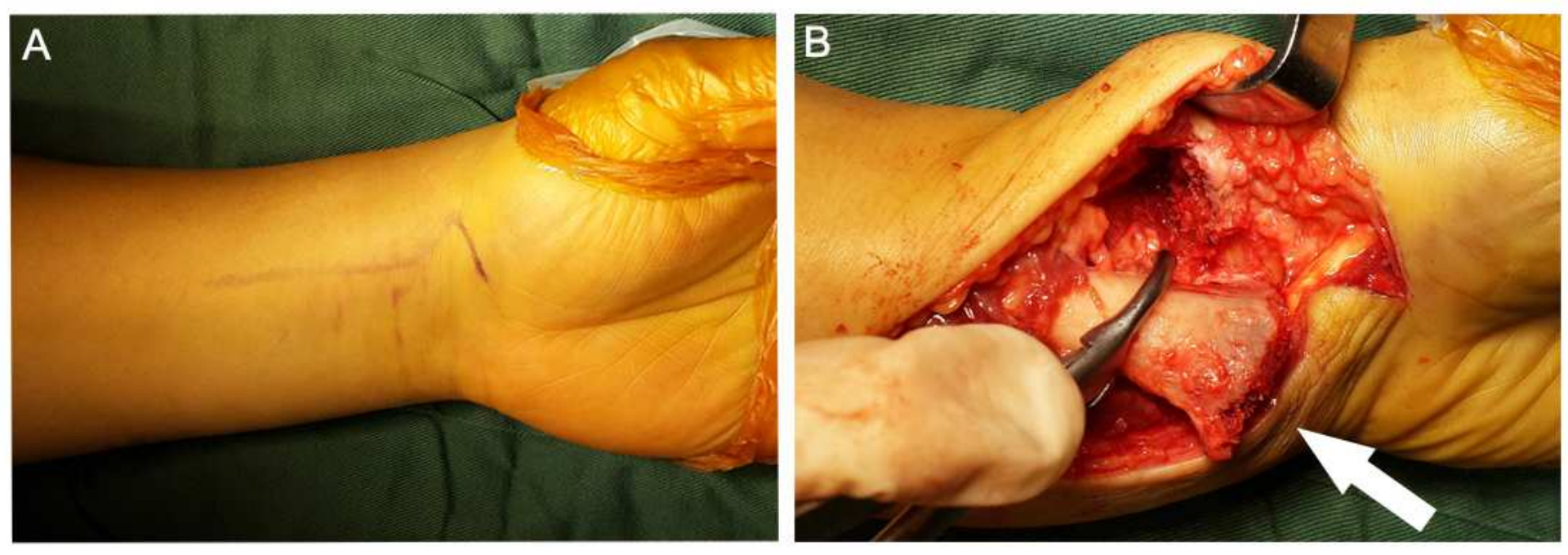

Figure 2

The incision and distal radius were reversed during the operation A) A zigzag incision was made across the wrist flexion crease to allow for better access and visualization. (B ) Intra focal exposure was obtained by pronating the proximal fragment out of the way with the turning radius (marked by the white arrow) using a bone
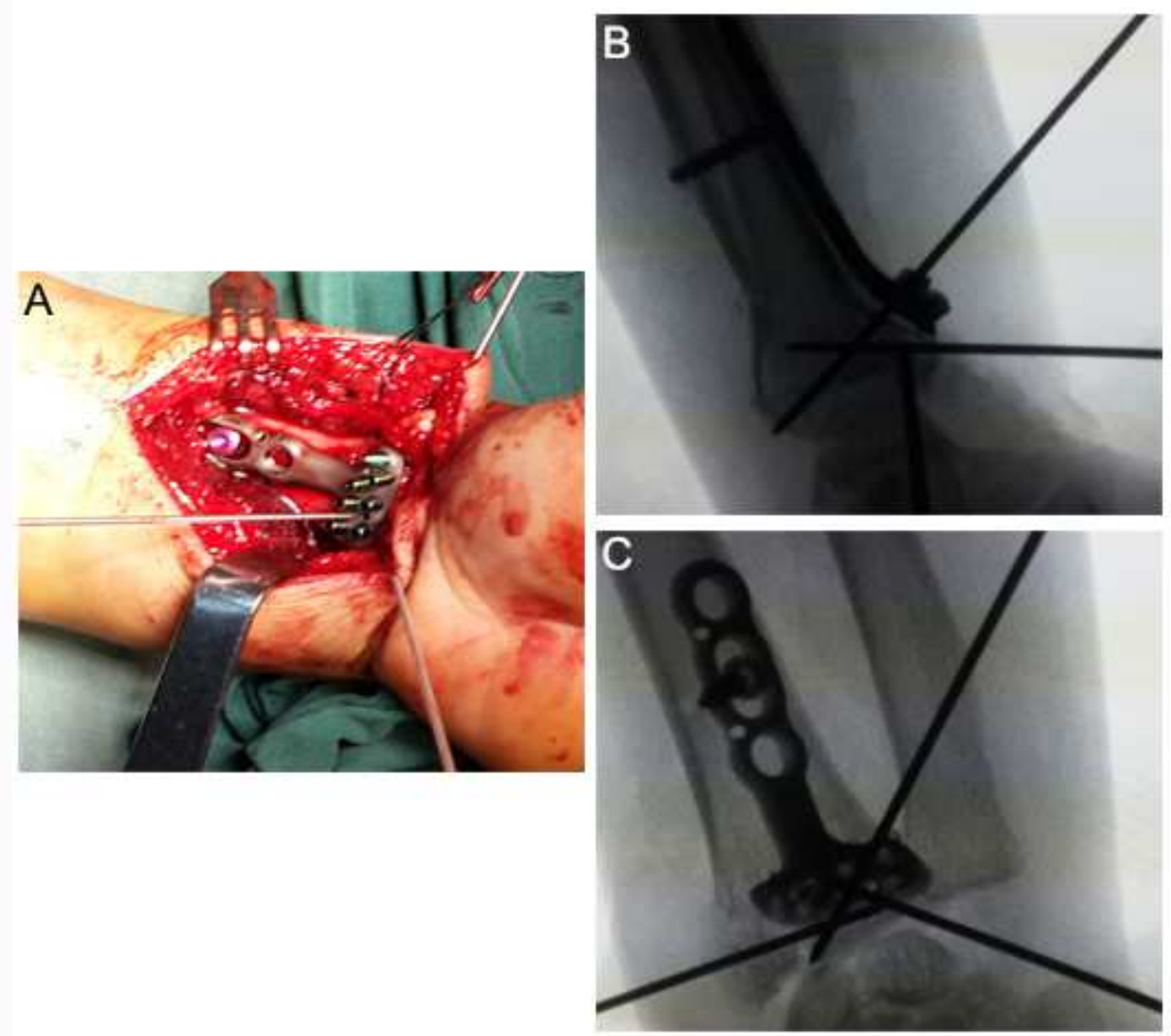

Figure 3 
Image of the operation and fluoroscopy observation. (A) Intraoperative image after the placement of DVR with $\mathrm{K}$ wires after open reduction of the fracture. $(B, C)$. Fracture temporally fixed with two $\mathrm{K}$ wires and plate temporally fixed with another K wire ( B , Lateral view; C , Anteroposterior view).
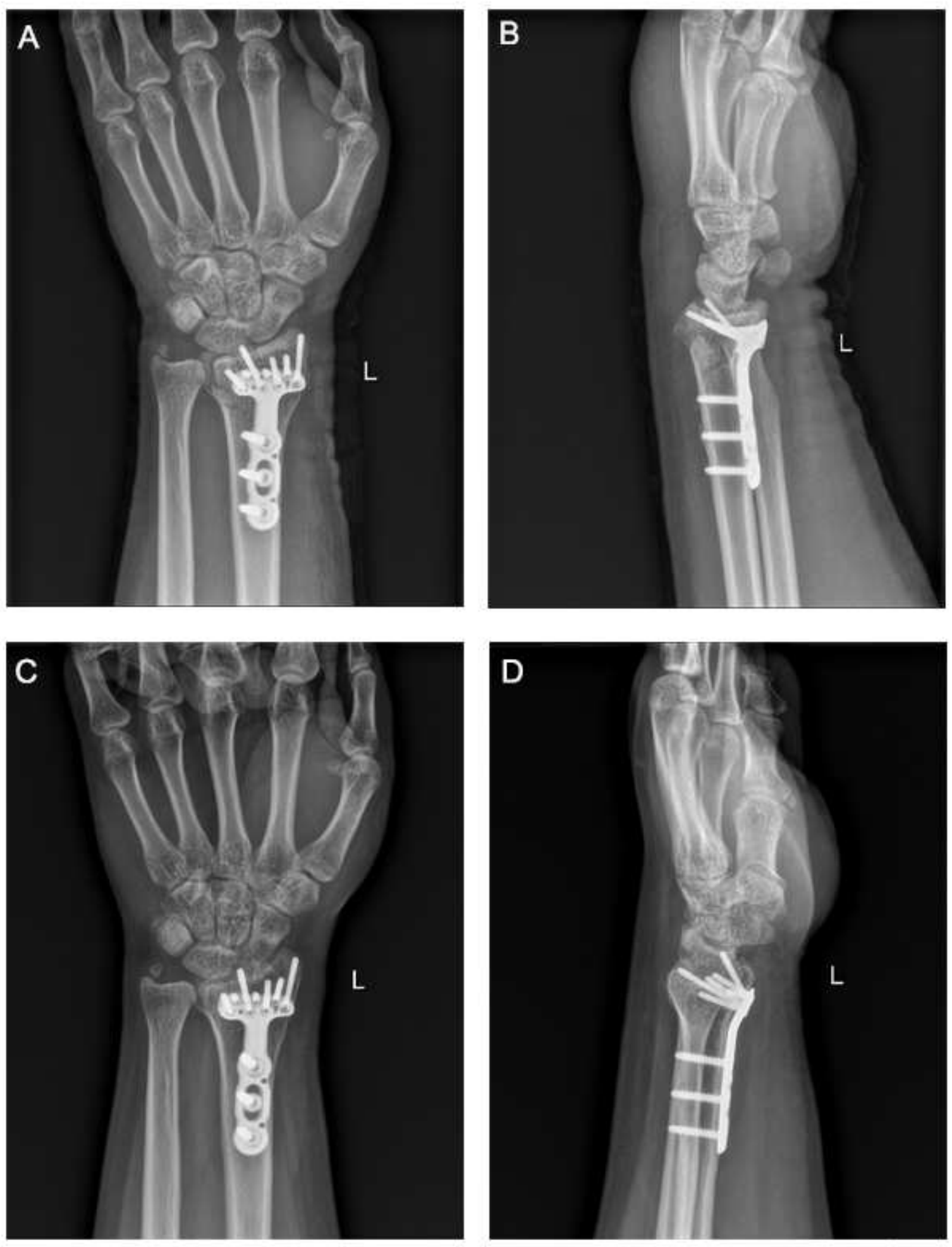

Figure 4

Radiographical images after the operation. ( A , B Anteroposterior A ) and lateral B) radiograph s showing that the alignment of the fracture and position of the plate were satisfactory on postoperative day 1 . ( $C$, 
D Anteroposterior C ) and lateral D ) radiograph s at twelve months post operation, showing that the left wrist has complete d bony union.
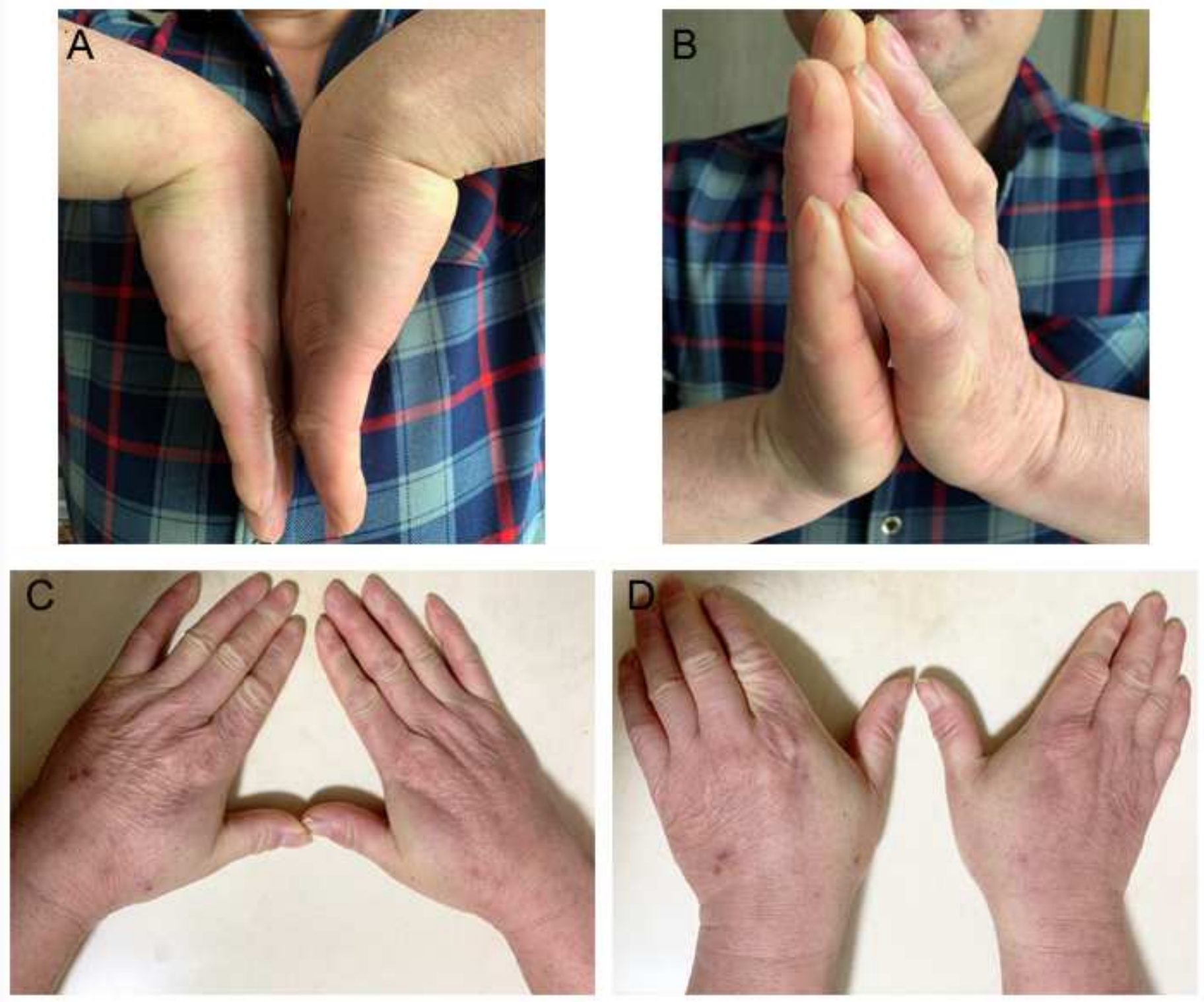

Figure 5

.Excellent clinical results 12 months after surgery. The patient achieved volar flexion of $77 \mathrm{~A}$ dorsal flexion of $80 \mathrm{~B})$ ), radial deviation of $20 \mathrm{C}$ ) and ulnar deviation of $23 \mathrm{D}$ 